\title{
Radio Polarimetry Results for Young Southern Pulsars
}

\author{
Fronefield Crawford, Victoria M. Kaspi \\ Center for Space Research, MIT, Cambridge, MA 02139, USA \\ Richard N. Manchester \\ ATNF, CSIRO, P.O. Box 76, Epping, NSW, Australia
}

\begin{abstract}
We present radio polarimetry results for nine Southern pulsars. Six of the nine are young, with characteristic ages less than 100 kyr and high spin-down luminosities. All six show significant linear polarization, and we confirm a previously noticed trend in which the degree of linear polarization increases with spin-down luminosity. We have used the rotating vector model to fit the observed position angle data for PSR J1513-5908 (B1509-58). We find that a magnetic inclination angle $\alpha>60^{\circ}$ is excluded at the $3 \sigma$ level in the fit, and that the geometry suggested by the morphology of an apparent bipolar X-ray outflow is marginally inconsistent with a recent model of the pulsar magnetosphere.
\end{abstract}

We present polarimetry results for nine Southern pulsars, six of which are young $\left(\tau_{c}<100 \mathrm{kyr}\right)$ with high spin-down luminosities $\left(\dot{E}>10^{34} \mathrm{erg} / \mathrm{s}\right)$. Our results complete the polarization information for the population of currently known young pulsars which are bright enough to be detected in radio polarization. All of the pulsars in our sample were observed at $1350 \mathrm{MHz}$ with the Parkes 64meter radio telescope, and in several cases were also observed at $660 \mathrm{MHz}$ and $2260 \mathrm{MHz}$. The hardware setup and observing technique are the same as those described elsewhere (Navarro 1994; Manchester, Han, \& Qiao 1998).

All six young pulsars show significant linear polarization at $1350 \mathrm{MHz}$ (PSRs J1105-6107, J1341-6220, J1513-5908, J1646-4346, J1730-3350, and J1801-2306). We confirm a previously noticed trend in which the degree of linear polarization is positively correlated with spin-down luminosity. This trend has been shown to be stronger at $4.9 \mathrm{GH} z$ (von Hoensbroech, Kijak, \& Krawczyk 1998 ) and is not evident at $400 \mathrm{MHz}$ (Gould \& Lyne 1998). Our results, which are concentrated at the high end of the $\dot{E}$ range in Fig. 1a, are consistent with this trend at $1350 \mathrm{MHz}$.

We have fit the rotating vector model of the position angle (PA) swing (Radhakrishnan \& Cooke 1969) to the $1350 \mathrm{MHz}$ data on PSR J1513-5908 (B1509-58). The PA swing is flat, consistent with emission coming from a partial conal beam (Manchester 1996). The polarization profile and PA swing are shown in Fig. 1b. From our PA fit we find a magnetic inclination angle range of $\alpha<60^{\circ}$ (3o confidence). Imposing the restriction that the angle between the spin axis and observer's line of sight is $\zeta z 70^{\circ}$, as proposed by Brazier \& Becker (1997) using the morphology of an apparent bipolar X-ray outflow, 

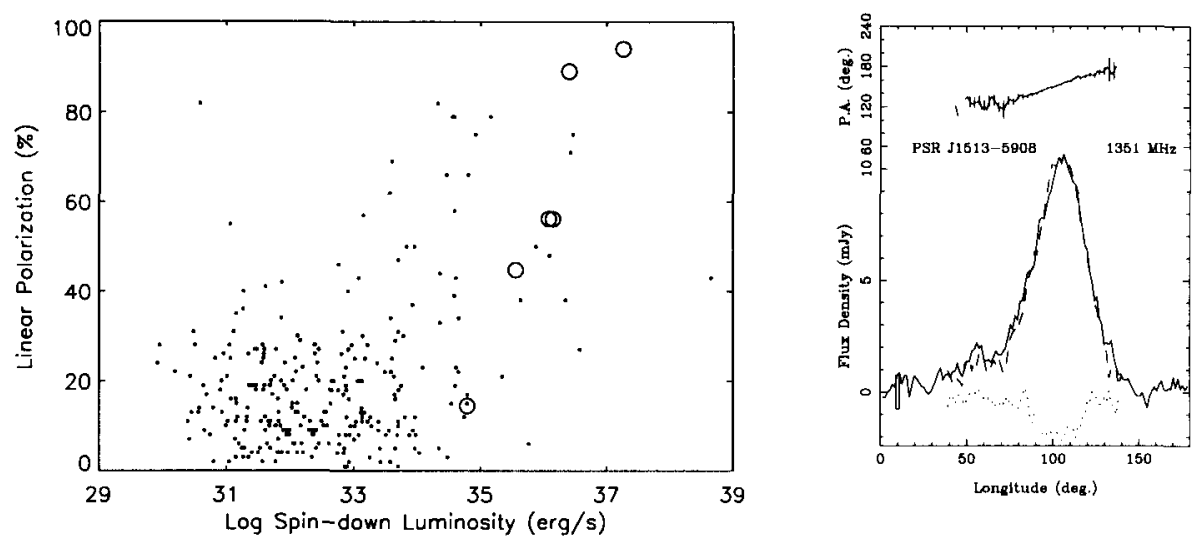

Figure 1. Left: Fractional linear polarization as a function of $\log \dot{E}$ for the six young pulsars in our sample at $1350 \mathrm{MHz}$ (open circles) and for 278 pulsars from Gould \& Lyne (1998) at $1400 \mathrm{MHz}$ (dots). Right: $1350 \mathrm{MHz}$ polarization profile for PSR J1513-5908 with position angle variation on top. The pulsar is almost completely linearly polarized.

implies $\alpha>30^{\circ}$ in our fit. In a model proposed by Melatos (1997) in which $P, \dot{P}$, and $\alpha$ uniquely determine the braking index $n$ of a pulsar, the range $\alpha>30^{\circ}$ for PSR J1513-5908 implies $n>2.86$. This is marginally inconsistent with the observed value of $n=2.837 \pm 0.001$ (Kaspi et al. 1994). The result from our fit indicates that the Melatos model and the geometry $\zeta z 70^{\circ}$ implied by Brazier \& Becker may be inconsistent with each other. The Melatos model may also be more directly tested using PSR J1513-5908 with the restriction $\alpha<60^{\circ}$ in combination with refinements in the spin parameters from future timing observations. We have submitted an article to the Astronomical Journal which details the results presented here.

\section{References}

Brazier, K. T. S. \& Becker, W. 1997, MNRAS, 284, 335

Gould, D. M. \& Lyne, A. G. 1998, MNRAS, 301, 235

Kaspi, V. M., Manchester, R. N., Siegman, B., et al. 1994, ApJ, 409, L57

Manchester, R. N. 1996, in Proc. of IAU Colloq. 160, 193

Manchester, R. N., Han, J. L., \& Qiao, G. J. 1998, MNRAS, 295, 280

Melatos, A. 1997, MNRAS, 288, 1049

Navarro, J. 1994, Ph.D. Thesis, California Institute of Technology

Radhakrishnan, V. \& Cooke, D. J. 1969, Astrophys. Lett., 3, 225

von Hoensbroech, A., Kijak, J., \& Krawczyk, A. 1998, A\&A, 334, 571 